\title{
Fears of COVID-19 and cancer recurrence related to work sustainability among male cancer survivors
}

\author{
Xiangyu Liu ${ }^{1}$, Andy SK Cheng ${ }^{2}$, Yingchun Zeng ${ }^{2}$, Xinqing Zhang ${ }^{3, \star}$, Xiaowei Peng ${ }^{4}$, \\ Huiping $\mathrm{Hu}^{4}$, Hua $\mathrm{Li}^{4}$, Michael Feuerstein ${ }^{5}$ \\ ${ }^{1}$ Center of Health Service, Hunan Cancer Hospital, Changsha, P. R. China \\ ${ }^{2}$ Department of Rehabilitation Sciences, The Hong Kong Polytechnic University, Hong Kong, P. R. China \\ ${ }^{3}$ School of Humanities and Social Sciences, Peking Union Medical College, Beijing, P. R. China \\ ${ }^{4}$ Department of Surgery, Hunan Cancer Hospital, Changsha, P. R. China \\ ${ }^{5}$ Uniformed Services University of the Health Sciences, Bethesda, USA
}

*Correspondence: xqzhang@pumc.edu.cn (Xin-Qing Zhang)

\begin{abstract}
Purpose: Male cancer survivors represent an important at-risk population for COVID-19. The COVID19 pandemic requires a global response for this most vulnerable population. This study purpose was to explore whether fear of COVID-19 and fear of cancer recurrence are related to the likelihood of remaining at work following treatment in male cancer survivors.

Survivors and methods: A cross-sectional survey was used. Data were collected in China in May to June 2020. The Fear of COVID-19 Scale, Fear of Cancer Recurrence (FCR) Inventory-Short Form and work sustainability subscale of the Readiness for Return to Work Scale were completed by male cancer survivors.

Results: A total of 121 employed male cancer survivors participated in this study. Fear of COVID-19 and fear of cancer recurrence were both negatively correlated with work sustainability $(\beta=-0.11$, and $\beta=-0.19$, respectively). Significant interaction effects between fear of COVID-19 and fear of cancer recurrence were observed $(\beta=0.46, P<0.01$ ). Advanced disease stage, undergoing radiation therapy and having recently completed cancer treatment were all factors related to lower work sustainability scores $\left(\beta=-0.28, \beta=-0.15\right.$, and $\beta=-0.17$, respectively). The overall path model yielded a good fit: $\chi^{2} / \mathrm{df}$ $=1.12(P=0.24)$, RMSEA $=0.07, \mathrm{TLI}=0.98, \mathrm{CFI}=0.99$, $\mathrm{IFI}=0.92$, and NFI $=0.96$.

Conclusion: Fear of COVID-19 is a mediator between fear of cancer recurrence and work sustainability among Chinese male cancer survivors. The findings also indicated that male cancer survivors with higher FCR levels reported less confidence in their ability to remain at work. This information can assist in the development of new interventions and educational programs for cancer survivors, healthcare providers and employers, to improve employees' ability to remain at work.
\end{abstract}

\section{Keywords}

Fear of COVID-19; Fear of cancer recurrence; Work retention; Male cancer survivors

\section{Introduction}

The COVID-19 pandemic has now spread to more than 200 countries/territories/regions [1]. The total number of confirmed COVID-19 cases worldwide now stands at more than 8.2 million, and nearly two million of deaths due to COVID-19 [2]. The COVID-19 pandemic has affected the world, particularly cancer survivors [3]. As cancer survivors represent an important at-risk population for COVID-19 [4], it is important to understand whether the fear of COVID-19 
is also related to work sustainability and whether it interacts with another fear, the fear of cancer recurrence, in male cancer survivors.

The relative five-year survival rate for all stages of male cancer is greater than $60 \%$ [5]. Consequently, there is a large and growing number of male cancer survivors [6]. But cancer diagnosis and treatment can impact survivors' ability to remain at work [7], as male cancer survivors report both long-term and late effects related to functional challenges [8]. Treatment effects occur concurrently with emotional responses, such as fear of cancer recurrence [9]. During the current pandemic, working cancer survivors may experience an additional emotional burden - fear of COVID-19 - especially as it relates to work - and work is a major functional goal for male cancer survivors [8].

There is an established association between fear of cancer recurrence and work sustainability in cancer survivors [10], and concerns related to infection and mortality associated with COVID-19 [11]. Therefore, this study aimed to examine both the common fear of cancer recurrence in male cancer survivors, and the timely fear of COVID-19, and the effects on male cancer survivors' motivation to remain at work.

\section{Survivors and methods}

\subsection{Study procedure}

The study was approved by the ethics committee of Hunan Cancer Hospital, The Affiliated Cancer Hospital of Xiangya School of Medicine, Central South University. All participants participated voluntarily in this study. Each survey question included the option of responding with, "I prefer not to answer", providing participants with control over their responses. All participants consented to participate in the survey.

\subsection{Study sample}

This study included adult men who were over 18 years of age, with a primary diagnosis of cancer across a spectrum of disease stages, who had completed major cancer treatment. Exclusion criteria were survivors who were younger than 18 years old, with cancer recurrence or who were still undergoing intensive cancer treatment.

\subsection{Measures}

\subsubsection{General information}

This study used a cross-sectional survey design. General information consisting of age, highest education level, employment status, marital status, disease stage, treatment types and time since primary cancer treatment completion, were collected using an online survey. In addition to the validated scale of fear of COVID-19, two questions related to its perceived impact - 'Does the COVID-19 pandemic contribute to your feelings of isolation?' and 'Does the COVID-19 pandemic have an impact on your cancer treatment protocols or follow-up plans?' - were collected.

\subsubsection{Fear of COVID-19 Scale (FCV-19S)}

The FCV-19S Chinese version was used to assess the level of fear of COVID-19 [12]. The FCV-19S is a seven-item scale in which items are answered using a five-point response format, with a total score ranging from 7 to 35 . Higher scores indicate greater levels of fear of COVID-19. A forward and backward translation was completed on a total of 75 male cancer survivors. The scale's measurement properties in the preliminary test of the measure's Chinese version had an internal consistency (Cronbach's alpha $)=0.94$. A preliminary exploratory factor analysis (EFA) was also examined. A maximum likelihood extraction method using varimax rotation was performed. The KMO was 0.88 and Bartlett's Test of Sphericity was significant $\left(\chi^{2}=1881.08, P<0.001\right)(\mathrm{n}=75)$. The EFA indicated that a one-factor solution accounted for $66.45 \%$ of the variance, with an eigenvalue of 4.65 . Factor loadings ranged from 0.58 to 0.73 . The FCV-19S floor and ceiling effects were acceptable, as the percentage of lowest and highest scores was less than $20 \%$.

\subsubsection{Fear of Cancer Recurrence Inventory (FCRI) - short form}

The FCRI Chinese version was used to assess the levels of fear of cancer recurrence. The FCRI short form was used. This is a nine-item scale answered using a five-point scale from 0 to 4 , with a total score ranging from 0 to 36. A higher score indicates greater levels of fear of cancer recurrence. An optimal cutoff score of 12 differentiates high fear of cancer recurrence from low fear of cancer recurrence [13]. In this study, the FCRI-9 Chinese version showed good internal consistency (Cronbach's alpha $=0.89$ ). The unitary factor structure was supported by the EFA, and the EFA revealed a single-factor structure, accounting for $59.29 \%$ of variance with an eigenvalue of 5.34, and factor loadings ranging from 0.56 to 0.80 .

\subsubsection{Readiness for Return to Work (RRTW) scale}

The Chinese version of the RRTW scale was used to assess motivation to remain at work or work sustainability. The full RRTW scale is a 22-item scale; however, this study only used the work maintenance subscale as a measure of work sustainability $[14,15]$, which consisted of a total of four items. Each item uses a five-point Likert scale, with " 1 " indicating strongly disagree and " 5 " indicating strongly agree. Higher scores indicate greater confidence in maintaining work. In this study, the work sustainability subscale showed good internal consistency, with Cronbach's alpha of 0.85 . The unitary factor structure was supported by the EFA for the subscale of work sustainability of RRTW, accounting for $69.36 \%$ with an eigenvalue of 2.77 , and factor loadings from 0.75 to 0.89 . The psychometric properties of these study measurement scales were summarized in Table 1.

\subsection{Data collection and analysis}

Data collection was conducted at the Hunan Cancer Hospital in Changsha, China, the provincial cancer hospital, from 
T A B L E 1. Reliability and validity of study measurement

\begin{tabular}{lcc}
\hline Scales & Reliability by Cronbach's alpha & Validity by exploratory factor analysis \\
\hline & & Ranging of item factor loading \\
Fear of COVID-19 Scale & 0.94 & $0.58-0.73$ \\
Fear of Cancer Recurrence Inventory - Short Form & 0.89 & $0.56-0.80$ \\
Readiness for Return-to-Work Scale & 0.85 & $0.75-0.89$ \\
\hline
\end{tabular}

May to June 2020. Data were analyzed using the Statistical Package for Social Sciences (SPSS) for Windows, version 25.0 (IBM Corp., Armonk, NY), and R-4.0.2. Descriptive statistics were summarized using SPSS. A path analytic approach using R portrayed the hypothesized "causal" paths between sociodemographic and clinical variables, fear of COVID-19, fear of cancer recurrence and work outcomes. The overall model fit was examined using goodness-of-fit indices: a nonsignificant Chi-square value $(P>0.05)$, normed fit index (NFI $\geq 0.90$ ), incremental fit index (IFI $\geq 0.90$ ), TuckerLewis index ( $T L I \geq 0.90$ ), comparative fit index (CFI $\geq 0.90$ ), and root mean square error of approximation (RMSEA $\leq$ $0.08)[16]$.

\section{Results}

All participants at the time of data collection were not COVID-19 patients and had no history of COVID-19 infection. Participant characteristics are reported in Table 2. The mean age for the study group was 38.22 years $(\mathrm{SD}=$ 9.12). A majority of cancer survivors reported at an early stage of disease $(\mathrm{n}=76,62.8 \%)$ and with cancer treatment of surgery only $(n=89,73.6 \%)$. During the data collection period, more than half of cancer survivors reported greater "feelings of isolation" than prior to COVID-19 ( $\mathrm{n}=74$, $61.2 \%$ ), and reported a delay in cancer treatment protocol implementation or delay in a follow-up plan due to the COVID-19 pandemic ( $n=72,59.5 \%)$.

The mean scores of fear of COVID-19, fear of cancer recurrence and work outcomes are listed in Table 3. Among these male cancer survivors, higher scores on fear of COVID19 included: "Feeling most afraid of COVID-19, and feeling uncomfortable thinking about COVID-19”. In terms of fear of cancer recurrence, a total mean score of FCRI was 11.97 (SD $=6.87)$, and $51.242 \%(\mathrm{n}=62)$ of cancer survivors reported a higher level of fear of cancer recurrence. Regression weights for each significant predictor variable are shown in Table 4. All significant study variables in the regression analysis were entered into the path analysis.

The overall path model (Fig. 1) yielded a good fit: $\chi^{2} / \mathrm{df}$ $=1.12(P=0.24)$, RMSEA $=0.07, \mathrm{TLI}=0.98, \mathrm{CFI}=0.99$, IFI $=0.92$, NFI $=0.96$. This model indicates there is a relationship between sociodemographic characteristics, clinical variables, fear of COVID-19, fear of cancer recurrence, and work sustainability. Importantly, there are significant interaction effects between fear of COVID-19 and fear of cancer recurrence $(\beta=0.46, P<0.01)$ as related to work sustainability. Both fear of COVID-19 and fear of cancer recurrence were inversely related to work sustainability ( $\beta$ $=-0.11$, and $\beta=-0.19$, respectively). Being at an advanced
T A B L E 2. Male cancer survivor characteristics $(N=121)$

\begin{tabular}{|c|c|c|c|}
\hline Variables & Mean (SD) & Range & $\mathbf{n}(\%)$ \\
\hline Age (years) & $38.22(9.12)$ & $19-58$ & \\
\hline \multicolumn{4}{|l|}{ Employment status } \\
\hline Working & & & $86(71.1)$ \\
\hline Sick leave & & & $35(28.9)$ \\
\hline \multicolumn{4}{|l|}{ Highest education } \\
\hline High school or below & & & $55(45.4)$ \\
\hline College or bachelor's degree & & & $60(49.6)$ \\
\hline Master's degree or above & & & $6(5.0)$ \\
\hline \multicolumn{4}{|l|}{ Marital status } \\
\hline Never married & & & $27(22.3)$ \\
\hline Married & & & $76(62.8)$ \\
\hline Divorced or widowed & & & $18(14.9)$ \\
\hline \multicolumn{4}{|l|}{ Cancer type } \\
\hline Thyroid cancer & & & $49(40.5)$ \\
\hline Prostate cancer & & & $35(28.9)$ \\
\hline Colorectal cancer & & & $37(30.6)$ \\
\hline \multicolumn{4}{|l|}{ Disease stage } \\
\hline Stage I & & & $76(62.8)$ \\
\hline Stage II & & & $39(32.2)$ \\
\hline Stage III & & & $6(5.0)$ \\
\hline \multicolumn{4}{|l|}{ Treatment type } \\
\hline Surgery & & & $89(73.6)$ \\
\hline Radiation therapy/chemotherapy & & & $18(14.9)$ \\
\hline Surgery + radiation + chemotherapy & & & $14(11.5)$ \\
\hline \multicolumn{4}{|l|}{ Feelings of isolation due to } \\
\hline \multicolumn{4}{|l|}{ COVID-19 pandemic } \\
\hline Less isolated than before & & & $4(3.3)$ \\
\hline The same as before & & & $43(35.5)$ \\
\hline More isolated than before & & & $74(61.2)$ \\
\hline \multicolumn{4}{|l|}{ Impact on any treatment protocol } \\
\hline \multicolumn{4}{|l|}{ or follow-up plans due to COVID-19 } \\
\hline No & & & $22(18.2)$ \\
\hline Yes & & & $72(59.5)$ \\
\hline Not yet, but worried & & & $27(22.3)$ \\
\hline Time since treatment completion & $13.75(13.04)$ & $0-84$ & \\
\hline
\end{tabular}

Abbreviation: COVID-19, coronavirus disease-2019.

disease stage, having undergone radiation therapy and having recently completed cancer treatment were all negatively associated with work sustainability $(\beta=-0.28, \beta=-0.15$, and $\beta=-0.17$, respectively). Feelings of isolation due to COVID19 were significantly associated with both the general fear of COVID-19 and fear of cancer recurrence $(\beta=0.29$, and $\beta=$ 0.14 , respectively). More recent treatment completion and impact on follow-up plans were also related to greater levels of fear of COVID-19 ( $\beta=-0.15, \beta=-0.38$, respectively). Age had an inverse relation to fear of cancer recurrence (i.e. younger age was related to higher levels of fear of cancer 
T A B L E 3. Fears and work sustainability $(N=121)$

\begin{tabular}{lcc}
\hline & Mean (SD)/n (\%) & Range \\
\hline Fear of COVID-19 & $16.71(6.38)$ & $7-35$ \\
Most afraid of COVID-19 & $3.02(1.18)$ & $1-5$ \\
Uncomfortable thinking about COVID-19 & $2.93(1.22)$ & $1-5$ \\
Hands become clammy when thinking about COVID-19 & $1.77(0.96)$ & $1-5$ \\
Afraid of losing life due to COVID-19 & $2.51(1.30)$ & $1-5$ \\
Become anxious when watching news about COVID-19 & $2.62(1.22)$ & $1-5$ \\
Cannot sleep due to worries about contracting COVID-19 & $1.80(0.99)$ & $1-5$ \\
Heart races or palpitates when thinking about COVID-19 & $2.03(1.17)$ & $1-5$ \\
Fear of Cancer Recurrence (FCR) & $11.97(6.87)$ & $0-36$ \\
Lower FCR (< 12) & $59(48.76)$ & $0-11$ \\
Higher FCR ( $\geq 12)$ & $62(51.24)$ & $12-36$ \\
Work sustainability & $12.15(3.80)$ & $4-20$ \\
\hline
\end{tabular}

T A B L E 4. Regression weights in fear of cancer recurrence, fear of COVID-19 and work sustainability $(\mathrm{N}=121)$

\begin{tabular}{llccccc}
\hline & & & Estimate & S.E. & C.R. & $P$ \\
\hline Fear of COVID-19 & $<-$ & Isolation feelings & 1.673 & 0.427 & 3.675 & $<0.001$ \\
Fear of COVID-19 & $<-$ & Impact on treatment & 0.074 & 0.033 & 2.348 & 0.019 \\
Fear of COVID-19 & $<-$ & Time since cancer treatment completion & -1.842 & 0.917 & -2.723 & 0.041 \\
FCR & $<-$ & Age & -0.092 & 0.029 & -2.321 & 0.021 \\
FCR & $<-$ & Disease stage & 3.021 & 0.765 & 2.098 & 0.018 \\
FCR & $<-$ & Isolation feelings & 1.945 & 0.964 & 2.675 & 0.032 \\
FCR & $<-$ & Fear of COVID-19 & 3.942 & 1.879 & 3.354 & 0.004 \\
& $<-$ & Treatment type & -0.126 & 0.052 & -2.467 & 0.011 \\
Work sustainability & $<-$ & Time since cancer treatment completion & -0.168 & 0.064 & -4.547 & $<0.001$ \\
Work sustainability & $<-$ & Fear of COVID-19 & -3.221 & 0.892 & -4.214 & $<0.001$ \\
Work sustainability & $<-$ & FCR & -2.251 & 0.823 & -2.025 & 0.013 \\
\hline
\end{tabular}

Abbreviations: CR, critical ratio; FCR, fear of cancer recurrence; SE, standard error.

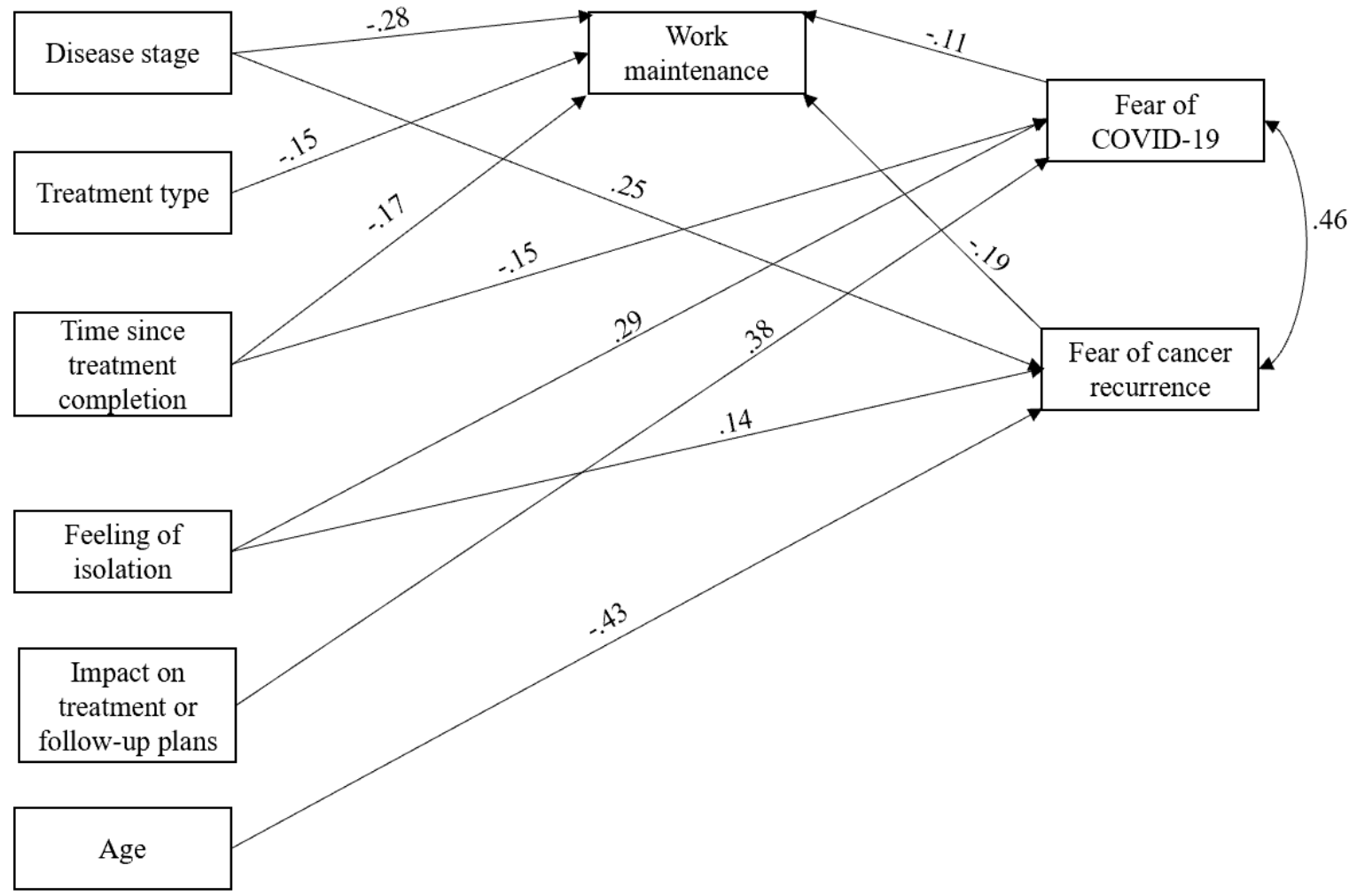

FIG. 1. Factors associated with work sustainability in male cancer survivors: path analysis. 
recurrence) $(\beta=-0.43)$. As expected, advanced disease stage was also related to greater fears of cancer recurrence $(\beta=$ $0.25)$.

\section{Discussion}

Despite being cancer survivors with relatively few residual problems [17], it was noted that nearly $50.82 \%$ of these male cancer survivors reported high levels of fear of cancer recurrence. Additionally, it is well known that the fear of cancer recurrence is one of most prevalent unmet needs in a homogeneous group of male cancer survivors [18]. Consistent with previous research [10, 18], younger age, advanced disease stage, and feelings of isolation were significant predictors of fear of cancer recurrence. In terms of work sustainability, advanced disease stage, radiation therapy and higher levels of fear of cancer recurrence were all related to lower confidence in work sustainability. This study also found that lower levels of fear of COVID-19 were related to more confidence in remaining at work.

This study's findings indicate that fear of COVID-19 is modestly associated with reduced work sustainability. As the pandemic has taken its toll on the global economy, members of the working-age population, especially male cancer survivors, may be more likely to lose their job or experience difficulties in finding a new job [4]. For example, those with a history of cancer can experience more problems in finding a new job compared to the general population [4, 19].

Adult cancer survivors remain an at-risk population during the COVID-19 pandemic [4]. Hence, healthcare providers must continue to distribute adequate information, including current concerns about COVID-19 on a range of topics, and its relationship with retention at work. This study's findings indicate that fear of COVID-19 was modestly related to higher levels of fear of cancer recurrence and lower confidence in the ability to maintain work. Therefore, oncologists, oncology nurses and healthcare providers of all types should determine the extent of these fears during follow-up surveillance visits [20]. They should help survivors allay their fears when necessary, as these fears are related to survivors' likelihood of remaining at work [21]. When these fears are reported at higher levels and are persistent, providers can facilitate relevant referrals in order to increase work sustainability [12, 22], an outcome that many survivors of various cancer types seek help for. Also, it is hoped that these findings will inform researchers and clinicians working on future interventions during the COVID-19 pandemic, and beyond that, will assist cancer survivors in remaining at work [23].

\subsection{Study limitations}

This study is not without limitations. This research was cross-sectional, which precludes any interference of causality or directionality. In addition, information on cancer stage was not independently validated through medical records, while all other clinical characteristics were confirmed by medical records and could be subject to recall bias. As knowledge sources and the housing conditions of the participants could be valuable and could affect the fear of COVID-19 [24], the lack of this data in this study could also be regarded as a limitation. Moreover, this study only included male cancer survivors, which may not representative all cancer survivors. Finally, while work sustainability can vary over time [4, 9, 19], and according to cancer types [25], future research should study more heterogeneous and representative groups in the cancer survivor population, using a longitudinal design matched with a non-cancer group.

\section{Conclusions}

The study found that male cancer survivors with higher levels of FCR reported lower confidence in work sustainability, and those with higher levels of both fears may have less confidence in their ability to remain at work. This study also revealed that fear of COVID-19 is a mediator between fear of cancer recurrence and work sustainability among male cancer survivors.

\section{Author contributions}

XZ, YZ: Funding acquisition, supervision, methodology, and writing. ASKC, XL, XP: Visualization, and editing. XL, XP: Data management and analysis. HH, HL: Data collection. XZ, XL, XP, MF: conceptualization, supervision, and writing-review and editing.

\section{Ethics approval and consent to participate}

This study was approved by Hunan Cancer Hospital's Ethical Committee.

\section{Acknowledgment}

Thanks for all participants jointed in this study.

\section{Funding}

This study was funded by Early Career Research Fund for doctorate level staff of The Third Affiliated Hospital of Guangzhou Medical University (\#2019B02); Hunan Provincial Department of Science and Technology (2018SK50909); and National Cancer Fund Center Project (NCC201818B62).

\section{Conflict of interest}

The authors have no conflicts of interest to declare.

\section{References}

1] World Health Organization. Coronavirus disease 2019 (COVID-19) situation report-209. 2020. Available at: https://www.who. int/docs/default-source/coronaviruse/situationreports/20200816-covid-19-sitrep-209.pdf?sfvrsn= 5dde1ca2_2 (Accessed: 1 September 2020). 
[2] Johns Hopkins University \& Medicine. COVID-19 Dashboard by the Center for Systems Science and Engineering (CSSE) at Johns Hopkins University (JHU). 2020. Available at: https : / / coronavirus . jhu . edu/map.html (Accessed: 31 December 2020).

[3] Printz C. When a global pandemic complicates cancer care. Cancer. 2020; 126: 3171-3173.

[4] Desai A, Sachdeva S, Parekh T, Desai R. COVID-19 and cancer: lessons from a pooled meta-analysis. JCO Global Oncology. 2020; 6: 557-559.

[5] Siegel RL, Miller KD, Jemal A. Cancer statistics, 2020. CA: A Cancer Journal for Clinicians. 2020; 70: 7-30.

[6] Wild CP, Weiderpass E, Stewart BW. World cancer report: cancer research for cancer prevention. Lyon, France: International Agency for Research on Cancer. 2020.

[7] Aschebrook-Kilfoy B, James B, Nagar S, Kaplan S, Seng V, Ahsan H, et al. Risk factors for decreased quality of life in thyroid cancer survivors: initial findings from the North American thyroid cancer survivorship study. Thyroid. 2015; 25:1313-1321.

[8] Diamond-Rossi SA, Jonklaas J, Jensen RE, Kuo C, Stearns S, Esposito $\mathrm{G}$, et al.Looking under the hood of "the Cadillac of cancers:" radioactive iodine-related craniofacial side effects among patients with thyroid cancer. Journal of Cancer Survivorship. 2020; 14: 847-857.

[9] Penedo FJ, Oswald LB, Kronenfeld JP, Garcia SF, Cella D, Yanez B. The increasing value of eHealth in the delivery of patient-centred cancer care. The Lancet Oncology. 2020; 21: e240-e251.

[10] Simard S, Thewes B, Humphris G, Dixon M, Hayden C, Mireskandari $S$, et al. Fear of cancer recurrence in adult cancer survivors: a systematic review of quantitative studies. Journal of Cancer Survivorship. 2013; 7: 300-322.

[11] Wang H, Zhang L. Risk of COVID-19 for survivors with cancer. The Lancet Oncology. 2020; 21: e181.

[12] Ahorsu DK, Lin CY, Imani V, Saffari M, Griffiths MD, Pakpour $\mathrm{AH}$. The fear of COVID-19 scale: development and initial validation. International Journal of Mental Health and Addiction. 2020; 1-9.

[13] Peng L, Huang W, Zhang W, Xu Y, Lu F, Zhong L, et al. Psychometric properties of the short form of the Fear of Cancer Recurrence Inventory (FCRI) in Chinese breast cancer survivors. Front Psychiatry. 2019; 10: 537.
[14] Park J, Roberts MR, Esmail S, Rayani F, Norris CM, Gross DP. Validation of the readiness for return-to-work scale in outpatient occupational rehabilitation in Canada. Journal of Occupational Rehabilitation. 2018; 28: 332-345.

[15] Xu YW, Luo XY, Lu XW, Lam SC. Reliability and validity of the lam assessment of employment readiness for injured workers. Chinese Journal of Rehabilitation Theory and Practice. 2014; 20: 592-596.

[16] Kline RB. Principles and practice of structural equation modeling. 2nd ed. New York: Guilford Press. 2005.

[17] Hedman C, Strang P, Djärv T, Widberg I, Lundgren CI. Anxiety and fear of recurrence despite a good prognosis: an interview study with differentiated thyroid cancer patients. Thyroid. 2017; 27: 1417-1423.

[18] Papaleontiou M, Reyes-Gastelum D, Gay BL, Ward KC, Hamilton AS, Hawley ST, et al. Worry in thyroid cancer survivors with a favorable prognosis. Thyroid. 2019; 29: 1080-1088.

[19] Nott D. The COVID-19 response for vulnerable people in places affected by conflict and humanitarian crises. The Lancet. 2020; 395: 1532-1533.

[20] Feuerstein M, Todd BL, Moskowitz MC, Bruns GL, Stoler MR, Nassif $\mathrm{T}$, et al. Work in cancer survivors: a model for practice and research. Journal of Cancer Survivorship. 2010; 4: 415-437.

[21] American Society of Clinical Oncology. A Guide to Cancer Care Delivery During the COVID-19 Pandemic. 2020. Available at: https://www. asco.org/asco-coronavirus-information (Accessed: 30 June 2020).

[22] Liang W, Guan W, Chen R, Wang W, Li J, Xu K, et al. Cancer patients in SARS-CoV-2 infection: a nationwide analysis in China. The Lancet Oncology. 2020; 21: 335-337.

[23] Shaw WS, Main CJ, Findley PA, Collie A, Kristman VL, Gross DP. Opening the workplace after COVID-19: what lessons can be learned from return-to-work research? Journal of Occupational Rehabilitation. 2020; 30: 299-302.

[24] Guven DC, Sahin TK, Aktepe OH, Yildirim HC, Aksoy S, Kilickap S. Perspectives, knowledge, and fears of cancer patients about COVID19. Frontiers in Oncology. 2020; 10: 1553.

[25] Ekenga CC, Kwon E, Kim B, Park S. Long-term employment outcomes among female cancer survivors. International Journal of Environmental Research and Public Health. 2020; 17: 2751. 